\title{
Bildwandlerbedienung, Strahlenschutz und Einweisung
}

\author{
Thomas Lein, Falko Moritz, Dirk Lehmann, Felix Bonnaire
}

\section{Zusammenfassung}

Der Einsatz eines Röntgenbildverstärkers ermöglicht die moderne operative Frakturversorgung. Dabei stehen die anzustrebende optimale intraoperative Bildgebung besonders mit dem Schutz des Patienten und des medizinischen Personals vor unerwünschter Strahlenexposition in Konkurrenz. Die Anwendung der bildgebenden Verfah-

\section{Einführung}

Wilhelm Conrad Röntgen entdeckte vor mehr als 100 Jahren die nach ihm benannten Strahlen, eine bahnbrechende Leistung, welche nicht nur die chirurgische Behandlung revolutionierte. Sie ermöglichte erstmals ohne Operation den Einblick in den menschlichen Körper zu diagnostischen Zwecken. Daraufhin setzte eine stürmische Entwicklung ein. Nach den ersten einfachen Röntgenaufnahmen und der Durchleuchtung unmittelbar am Leuchtschirm wurde die FilmFolien-Technik entwickelt. Einen weiteren Fortschritt stellte dann der Einzug des Röntgens in den Operationssaal dar, dieses erfolgte etwa 1930. Zunächst waren hier nur statische Aufnahmen mit einer mobilen Strahlenquelle möglich, die angefertigten Folienaufnahmen mussten in der Dunkelkammer entwickelt werden. Der Zeitaufwand war nicht unerheblich und die Aufnahme erst nach erfolgter Osteosynthese sinnvoll.

Durch die Entwicklung des Röntgenbildverstärkers und seinem breiten operativen Einsatz war der Chirurg in der Lage, Röntgenbilder selbst anzufertigen und sein operatives Vorgehen unmittelbar am Bildwandler zu kontrollieren.

OP-JOURNAL 2005; 21: 16-21

(c) Georg Thieme Verlag KG Stuttgart · New York ren im Operationstrakt unterliegt deshalb strengen gesetzlichen Richtlinien, welche einzuhalten sind. Durch Schulung der Mitarbeiter, geeignete Schutzmaßnahmen und eine exakte Planung des operativen Eingriffes ist es möglich, die Rate an unerwünschter und vielleicht schädigender Streustrahlung zu minimieren sowie die Bildgebung und damit die Versorgungssicherheit zu verbessern.
Der Einsatz von Computertomographie und Bildverstärkern mit dreidimensionaler Darstellung des Röntgenobjektes ermöglichen zum jetzigen Zeitpunkt moderne Operationsverfahren, dienen der Qualitätsverbesserung und erlauben minimalinvasives Vorgehen (Abb.1). Durch die digitale Bildverarbeitung und Bildnachbearbeitung, Halte- und Speicherfunktionen sowie die Netzwerkfähigkeit der neueren Geräte wird eine Qualitätssicherung und Bilddokumentation auf hohem Niveau ermöglicht.

\section{Wirkung ionisierender Strahlung}

Ionisierende Strahlen sind prinzipiell in der Lage, biologische Reaktionen respek- tive negative Wirkungen im Organismus zu bewirken (Tab.1). Dabei können deterministische von stochastischen Wirkungen abgegrenzt werden [6].

Deterministische (nichtstochastische) Wirkungen sind im Ausmaß von der Höhe der Dosis abhängig.

Es handelt sich hierbei um Wirkungen, die bei höheren Dosen zustande kommen und mit Symptomen der Strahlenkrankheit (Hautrötung, Haarausfall, gastrointestinale Beschwerden, Agranulozytose etc.) einhergehen. Da es eine direkte Beziehung zwischen der eingestrahlten Dosis und dem Ausmaß der Wirkung gibt, müssen diese Wirkungen nicht mithilfe der Wahrscheinlichkeitslehre (Stocha-

Tab. 1 Allgemeine Wirkungen ionisierender Strahlen
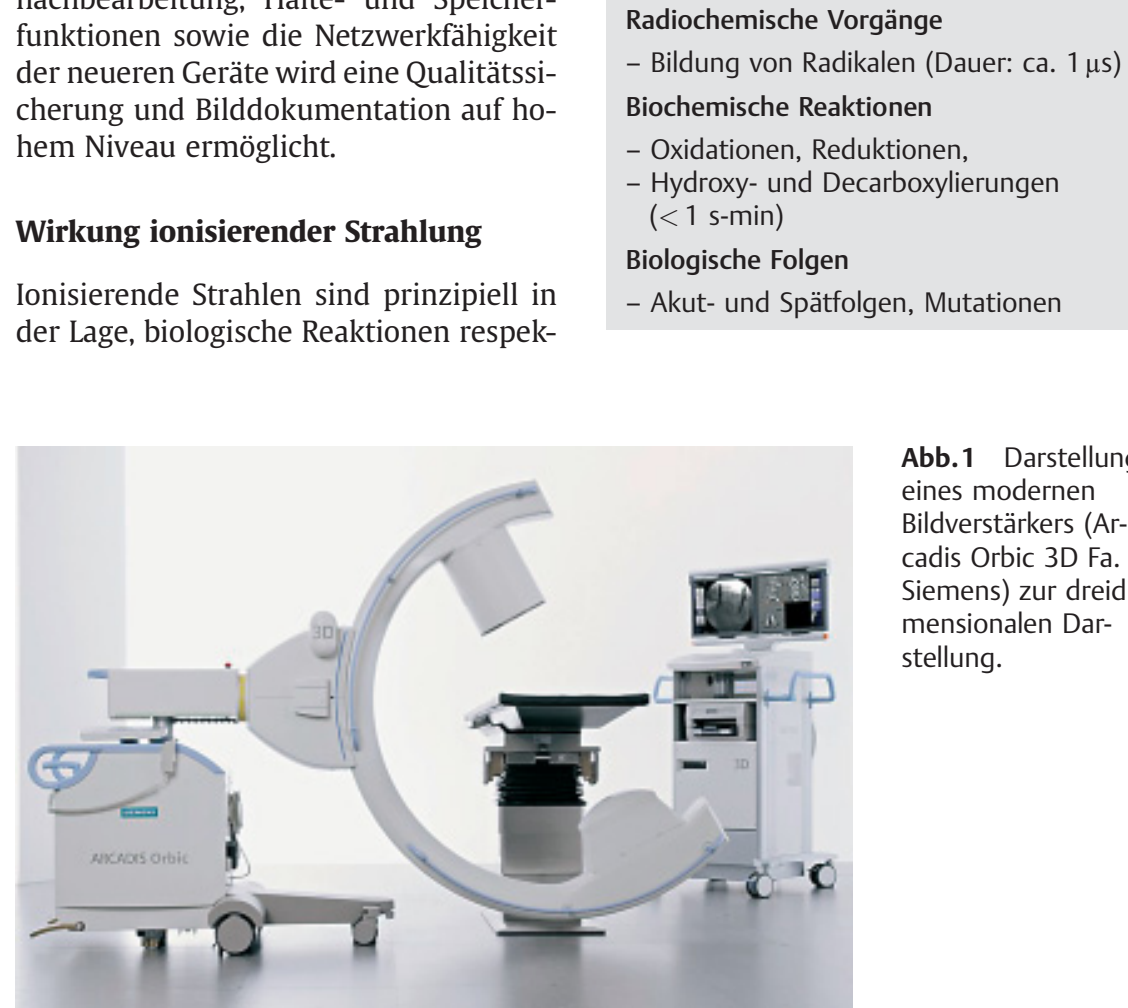

Abb. 1 Darstellung eines modernen Bildverstärkers (Arcadis Orbic 3D Fa. Siemens) zur dreidimensionalen Darstellung. 
stik) erfasst werden. Die dafür notwendigen Strahlendosen können heute aufgrund baulicher und gerätetechnischer Schutzvorkehrungen praktisch nicht mehr erreicht werden.

Stochastische Wirkungen kommen dagegen durch niedrige Dosen zustande.

Es handelt sich hierbei um Veränderungen des Erbgutes und die Induktion von Krebserkrankungen durch ionisierende Strahlen. Anders als bei deterministischen Wirkungen nimmt hier nur die Wahrscheinlichkeit von Schädigungen mit der Dosis zu, daß Ausmaß der Wirkung hat jedoch keinen Bezug zur auslösenden Dosis. Da nur mithilfe der Wahrscheinlichkeitsrechnungen „etwaige Wirkungen nicht auszuschließen sind oder wahrscheinlich sind“ sprechen wir von stochastischen Wirkungen (direkte Strahlenwirkung = Treffer: $\quad$ DNA-Schaden; indirekte Strahlenwirkung $=\mathrm{H}_{2} \mathrm{O}$ Radiolyse: DNA-Schaden).

\section{Einsatz des Bildwandlers im OP}

Moderne operative Frakturbehandlung vermittels Osteosynthese wird durch den Einsatz chirurgischer Röntgenbildverstärker erst ermöglicht und praktikabel.

Dabei sollten bereits präoperativ anhand entsprechender OP-Standards Einsatz, Aufbau und Stellung des Bildwandlers und des Monitors in Beziehung zu Operationstisch bzw. Unfallchirurg festgelegt sein (Abb. 2).

Hierbei sollen ergonomische Kriterien berücksichtigt werden: Der Operateur soll möglichst ohne Behinderung gerade antegrad den Monitor im Blickfeld haben und durch das Einschieben des Bildverstärkers nicht behindert werden. Intraoperative Stellungswechsel der Bildverstärkereinheit bringen immer Unruhe in das operative Geschehen, nicht selten gefährden sie die Sterilitätsnormen während eines chirurgischen Eingriffes.

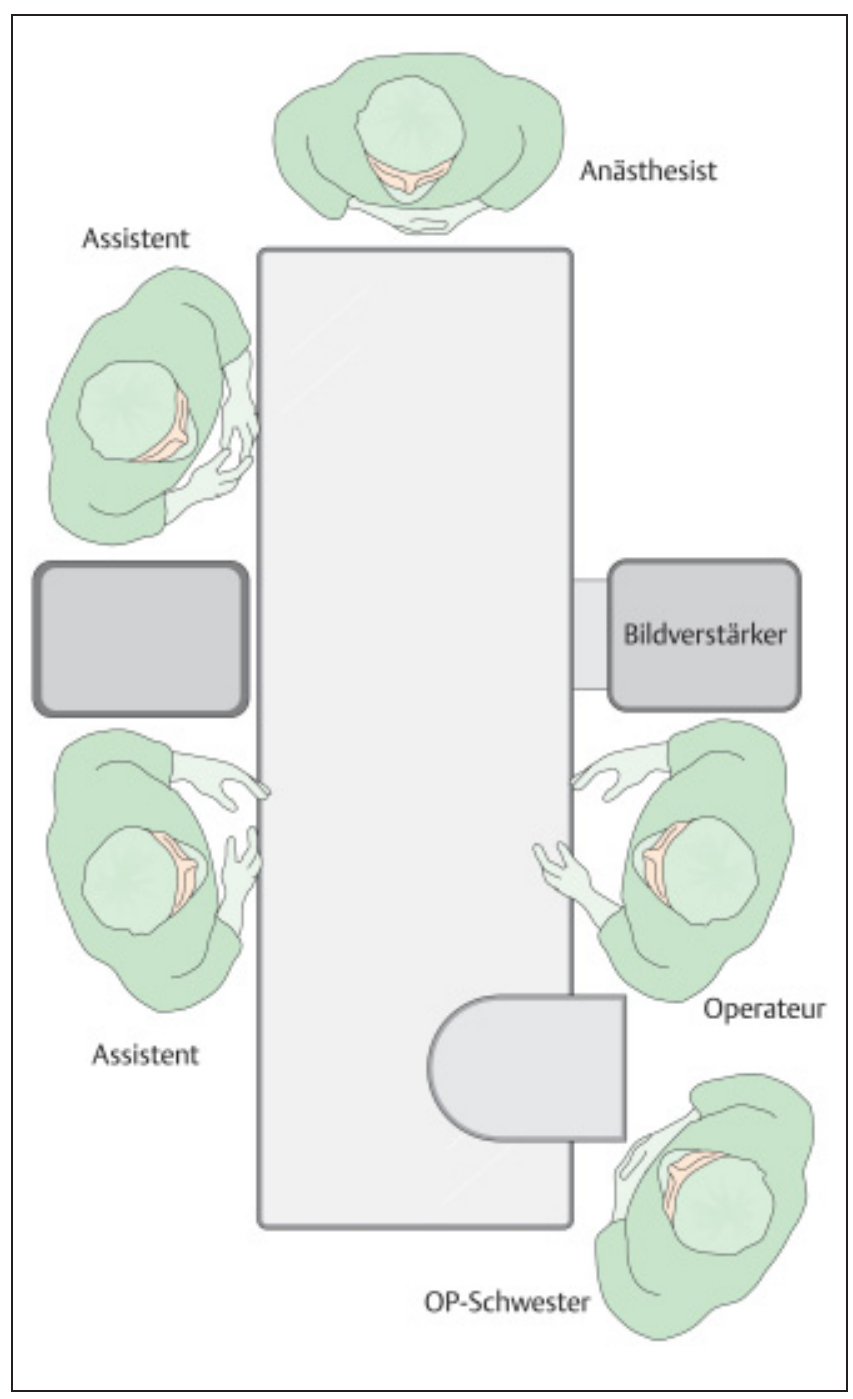

Abb. 2 Beispiel präoperative Planung-Stellung OPPersonal und Bildverstärker: 1: Operateur/2: Assistent/ 3: Assistent/4: OPSchwester/5: Anästhesist.
Die den Bildverstärker bedienende Person muss mit den technischen und apparativen Gegebenheiten des Gerätes und der Anatomie bekannt sein, um unnötige Fehlpositionierungen und damit fehlerhafte Röntgenaufnahmen zu minimieren.

Vorteilhaft ist das Bedienen durch eine RTA, alternativ können Arzt oder unsterile OP-Schwester den Bildverstärker bedienen, insofern sie unterwiesen worden sind. Vor dem Eingriff werden alle relevanten Daten über Patient, Operationsteam und Eingriff in das BV-Modul eingegeben, um die Bilddokumentation und Qualitätssicherung zu gewährleisten. Weiterhin muss sich der Operateur vor Beginn des Eingriffes von der Gerätefunktion überzeugen, um während der Operation Wartezeiten zu verringern.

Zusätzlich besteht die Pflicht zur Dokumentation der intraoperativen Röntgenleistung hinsichtlich Stärke und Dauer der Anwendung im vorgegebenen Operationsgebiet.

Von erheblichem Vorteil für die intraoperative Frakturversorgung unter Bildverstärker ist der Einsatz eines strahlendurchlässigen Operationstisches (z.B. Karbontisch der Fa. MAQUET). Hier können Artefakte und Überlagerungen durch Teile des Tisches ausgeschlossen werden, weiterhin ist eine Minimierung der Strahlendosis möglich, da nur Körperteile des Patienten im Strahlengang als Durchleuchtungsobjekt vorliegen [1].

\section{Intraoperativer Strahlenschutz}

Der Strahlenschutz des Patienten ist in der geltenden Röntgenverordnung (RöV) [7] in drei Bereichen geregelt:

- Es finden sich Hinweise zur Indikation in Form allgemeiner Bestimmungen.

- Die Qualifikationsvoraussetzungen derjenigen Personen, die Röntgenstrahlen anwenden, sind dagegen präzise durch eine Fachkunderichtlinie festgelegt. Sowohl die anordnenden Ärzte als auch das technische Personal müssen fachkundig im Sinne der RöV sein.

- Die Röntgentechnik schließlich unterliegt ebenfalls strengen Bestimmungen in Form von Bauartzulassungen, Abnahme- und Konstanzprüfungen. Hinzu kommen Stichproben durch die mit der Qualitätssicherung befassten Organe der Ärztekammern und Kassenärztlichen Vereinigungen. 
Tab. 2 Strahlenschutzregeln beim Betrieb eines Röntgenbildverstärkers (nach Klimpel) [5]

1. Kurze Durchleuchtungszeit

2. Interruptschaltung bei Durchleuchtung

3. Nutzstrahlfeld einblenden

4. Abstand des Operateurs zu Nutzstrahlfeld und Patienten groß halten

5. Im seitlichen Strahlengang steht der Operateur hinter dem BV

6. Optimale Strahlenschutzkleidung tragen

7. Durchleuchtung erst bei richtiger Position $\mathrm{BV}$ beginnen

8. OP-Team muss mit der gesamten technischen Ausstattung vertraut sein

Intraoperativ muss dem Strahlenschutz dem Patienten gegenüber als auch dem Schutz des medizinischen Personals Rechnung getragen werden. Die bei der Röntgendurchleuchtung auftreffende Nutzstrahlung durchdringt den Patientenkörper zum Teil, gelangt als Strahlenrelief zum Bildempfängersystem und dient der Bildgebung. Ein anderer Teil der Strahlen wird absorbiert, ein dritter im Patientenkörper gestreut (ComptonStreuung) und tritt in Form energieärmerer, so genannter Streustrahlung allseits aus dem Körper des Patienten aus.

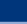
Da mobile Röntgengeräte wegen ihrer besonderen Anwendung über keine geräteseitigen Abschirmungen verfügen, sollte das Operationsteam den Strahlenschutz besonders beachten [3].

Hierzu fordert die RöV eine Vielzahl von Maßnahmen zur Verringerung der Strahlenbelastung, wesentliche Strahlenschutzregeln zielen auf die niedrigste zu erreichende Exposition von Patient und medizinischem Personal ab (Tab.2).

Für den so genannten Kontrollbereich (Tab.3) gelten nach RöV §21 Bestimmungen zum Tragen der Schutzkleidung und nach $\S 35$ zum Messen der Körperdosis [7]. Der Strahlenschutz für das Operationsteam wird intraoperativ durch das Tragen einer Operationsschürze im Bleigleichwert

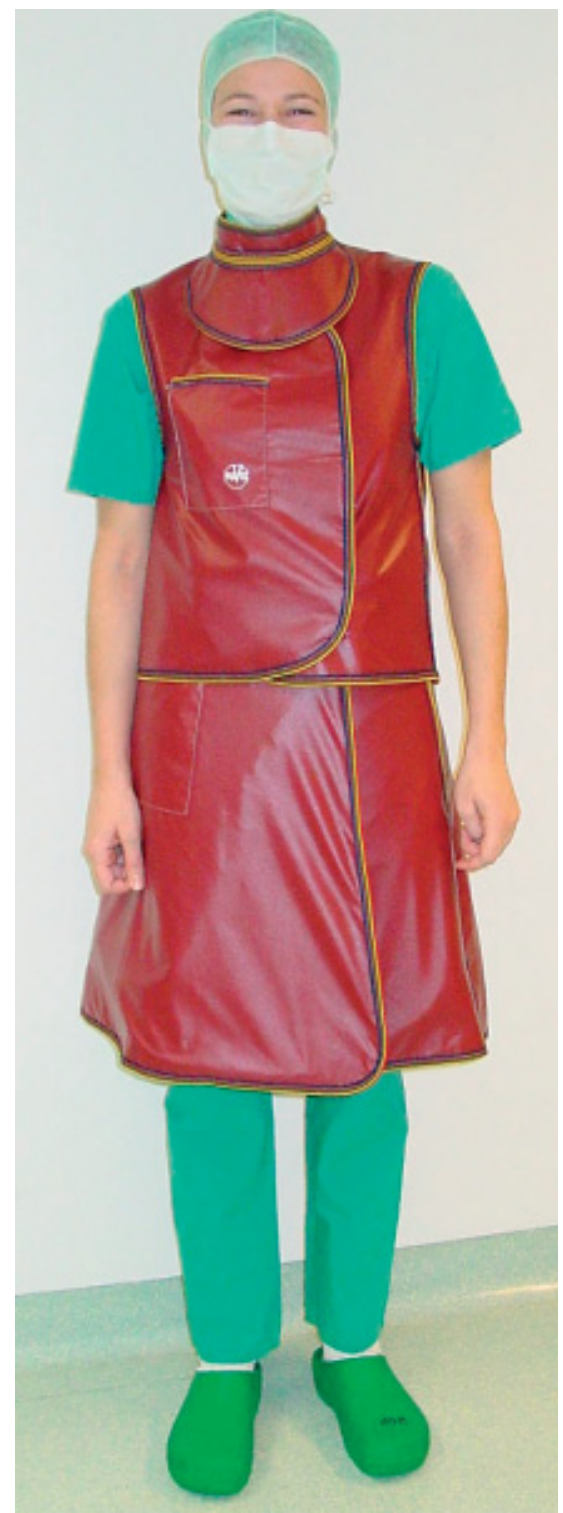

Abb. 3 Tragen der Röntgenschutzkleidung.

von 0,25 mm, dem Schilddrüsenschutz im Bleigleichwert $0,5 \mathrm{~mm}$ und falls notwendig mit Strahlenschutzhandschuhen mindestens $35 \mathrm{~cm}$ lang - sowie ortsveränderlichen Abschirmungen optimiert (Abb.3). Es besteht die Pflicht zum Tragen eines Personendosimeters, dieses sollte immer in gleicher Höhe, vorteilhaft über der linken Brust unter der Röntgenschutz-

Tab. 3 Kontrollbereiche beim Bildwandlereinsatz

\begin{tabular}{lll} 
& \multicolumn{2}{c}{$\begin{array}{c}\text { Strahlenschutzbereiche für mobile Röntgengeräte } \\
\text { Kontrollbereich }\end{array}$} \\
\hline Mobilet & bis $1,50 \mathrm{~m}$ & ab $1,50 \mathrm{~m}$ \\
C-Bogen & bis $4,00 \mathrm{~m}$ & ab $4,00 \mathrm{~m}$ \\
\hline
\end{tabular}

$\measuredangle$ Strahlenbelastung im Kontrollbereich sollte nur durch Streustrahlung entstehen!

$\measuredangle$ Bester Strahlenschutz ist der Abstand!

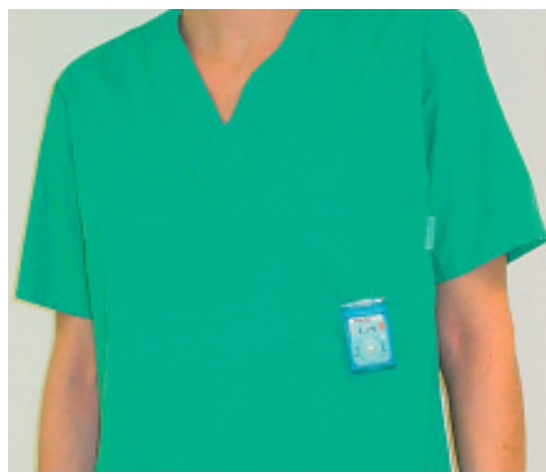

Abb. 4 Tragen des Personendosimeters.

kleidung getragen werden (Abb.4). Die Personendosimeter werden im Regelfall monatlich durch die Abteilung Dosimetrie oder eine andere Prüfstelle ausgewertet.

Zutritt zum Kontrollbereich haben exakt definierte Personen (Tab.4), weiterhin muss die Durchführung intraoperativer Röntgenstrahlung bei geschlossenen Türen und dem sichtbaren Vermerk: ACHTUNG RÖNTGEN - KEIN ZUTRITT! erfolgen.

Der technische, nicht administrative Strahlenschutz basiert auf drei Maßnahmen:

1. Abstand (möglichst weit entfernt vom Strahlengang)

2. Bestrahlungszeit (so kurz wie möglich)

3. Abschirmung (Schutzkleidung und abschattende Objekte nutzen).

Nach dem Abstandsquadratgesetz nimmt die Strahlendosis nicht linear, sondern mit dem Quadrat des Abstandes von der Strahlenquelle ab. Somit ist durch Vergrößerung der Distanz zum Nutzstrahlenfeld eine deutliche Minimierung der Exposition möglich. Messungen an

Tab. 4 Zugelassene Personen im Kontrollbereich

\section{Zutritt Kontrollbereich}

- Personal, das zur Durchführung der Untersuchung erforderlich ist

- Auszubildende über 18 Jahre

- Auszubildende unter 18 Jahren mit behördlicher Genehmigung

- zu untersuchender Patient

- helfende Person - freiwillig unterstützende Person

$\checkmark$ sind über mögliche Gefahren und deren Vermeidung zu unterweisen

$\measuredangle$ sofort ablesbare Dosimeter 

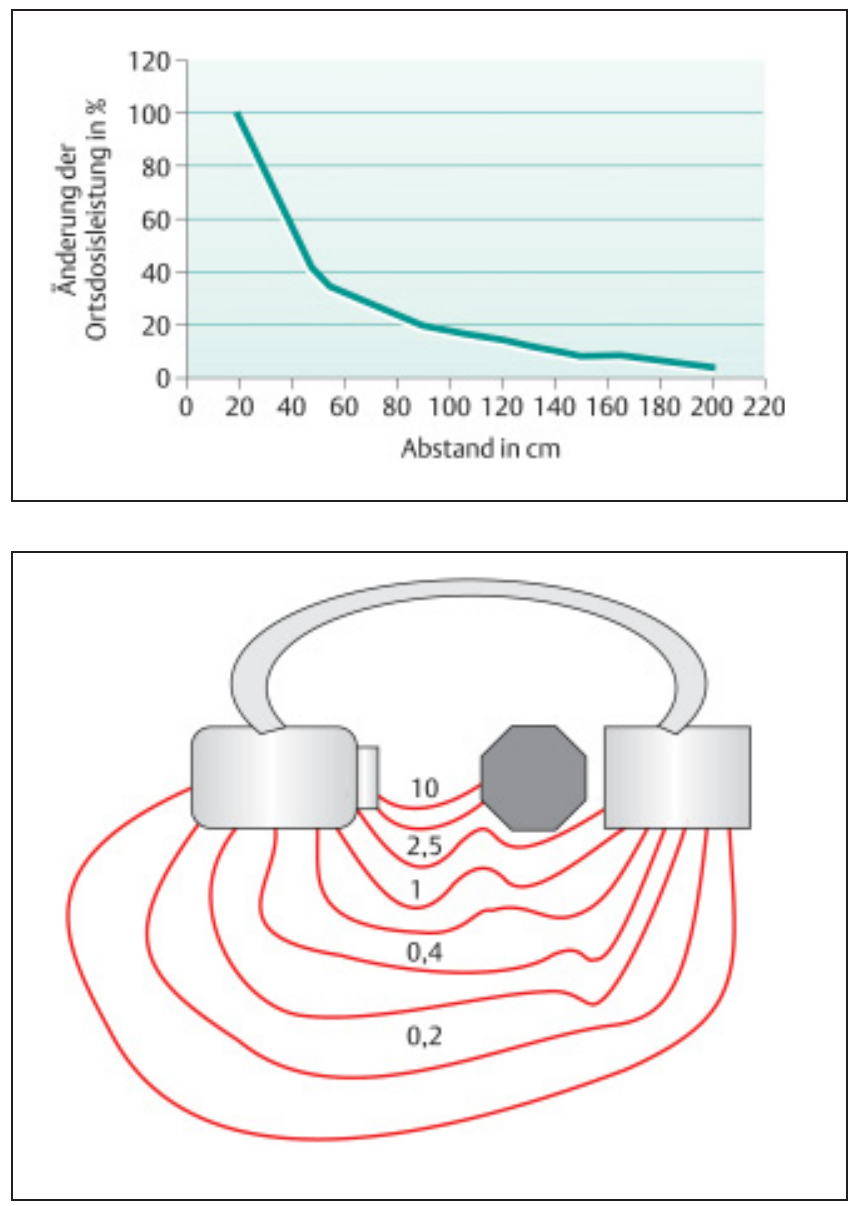

einem Phantom zur Abnahme der Dosisleistung zeigten deutlich die Bedeutung des Abstandes von der Strahlenquelle insbesondere im Nahbereich unter einem Meter (Abb. 5) [2].

Die Durchleuchtungszeit sollte insbesondere im seitlichen Strahlengang kurz gehalten werden, da hier eine höhere Streustrahlenexposition auftritt als im posterior-anterioren Strahlengang.

Der Austritt der Streustrahlung aus dem Patienten weist ein Maximum schräg seitlich in Richtung zum Röntgenstrahler auf und führt zu einem typischen Isodosen-Verlaufsmuster um den Bildverstärker (Abb.6). Weiterhin von Vorteil ist die Anordnung des C-Bogens mit dem Strahler unter dem Operationstisch, da die strahlensensiblen Organe im Kopfbereich des Operateurs (Augen, Schilddrüse) hier zusätzlich geschützt werden. Auch bei der Durchführung von Osteosynthesen sind durch geeignete Zielgeräte, Repositionshilfen bzw. Verbesserung und Vereinfachung von Operationstechniken positive Dosisreduktionen weiterhin möglich.
Abb. 6 Darstellung der typischen Isodosenverteilung um einen Bildverstärker [2].

Abb.5 Abnahme der Dosisleistung der Streustrahlung senkrecht zum Strahlengang neben einem C-Bogen mit wachsendem Abstand vom Patienten (nach Fuchs) [2].

\section{Einweisung des Personals}

Strahlenschutzverantwortlicher ist, wer eine Röntgeneinrichtung betreibt.

In einem Krankenhaus ist das der Krankenhausträger, welcher in der Regel die Chefärzte der Abteilungen, in denen Röntgenstrahlen angewendet werden, zu Strahlenschutzbeauftragten bestellt. Dabei ist ein wichtiger Aspekt, dass der Strahlenschutzbeauftragte nur für den Bereich bestellt werden kann, in welchem er weisungsberechtigt ist. So kann ein Chefarzt der Radiologischen Abteilung nicht als Strahlenschutzbeauftragter im chirurgischen Operationssaal fungieren. Aufgabe des Strahlenschutzbeauftragten ist es unter anderem, dem Strahlenschutzverantwortlichen unverzüglich alle Mängel mitzuteilen, die den Strahlenschutz beeinträchtigen.

Ärzte und Pflegepersonal gelten als „beruflich strahlenexponierte Personen“ wenn sie in ihrem Tätigkeitsbereich einer Strahlenexposition ausgesetzt sind, durch welche sie einer jährlichen Ganzkörperdosis ab 5 mSv oder einer entspre- chend höheren Teilkörperdosis ausgesetzt werden können.

Dabei wird die Kategorie A mit effektivem Dosisgrenzwert für Keimdrüsen, Gebärmutter und rotem Knochenmark bei $50 \mathrm{mSv} / \mathrm{Jahr}$ von exponierten Personen der Kategorie B unterschieden. Personen der Kategorie A müssen in mindestens jährlichen Abständen durch einen hierfür ermächtigten Arzt einer Strahlenschutzuntersuchung zugeführt werden.

Personen, denen der Zutritt zum Kontrollbereich nach $\S 22$, Abs.1 der RöV erlaubt ist, müssen halbjährlich durch den Strahlenschutzbeauftragten respektive den Strahlenschutzverantwortlichen belehrt werden. Inhalt der Belehrungen sind Arbeitsmethoden, die möglichen Gefahren, die anzuwendenden Schutzmaßnahmen und der für die Tätigkeit wesentliche Inhalt von Röntgenverordnungen und Genehmigungen. Über die durchgeführte Belehrung besteht Dokumentationspflicht.

\section{Fazit}

Der Einsatz eines modernen Röntgenbildverstärkersystems ermöglicht intraoperativ die „online“ Frakturversorgung bei sehr guter Bildinformation. Daher ist der Einsatz von Röntgenstrahlen in der Versorgung des traumatisierten Patienten nicht mehr wegzudenken. An der Verbesserung der bildgebenden Systeme wird gearbeitet und es finden sich seit 2000 Röntgensysteme mit dreidimensionaler Bildgebung in der Anwendung (z.B. ISO $\mathrm{C}^{3}$ und Arcadis Orbic 3D, Fa. Siemens). Dies bedeutet einen weiteren Fortschritt in der Ergebnisverbesserung, dem Trend zu minimalinvasiven Verfahren und einer optimalen Dokumentationssicherheit und -qualität [8].

Darüber hinaus stehen weiterhin intraoperativ besonders der Schutz des Patienten und des medizinischen Personals vor unerwünschter Strahlenexposition im Vordergrund.

Die Anwendung der bildgebenden Verfahren im Operationstrakt unterliegt strengen gesetzlichen Richtlinien, welche unbedingt einzuhalten sind.

Durch regelmäßige Schulung der Mitarbeiter, geeignete Schutzmaßnahmen und eine exakte Planung des operativen Eingriffes ist es möglich, weiterhin die Rate an unerwünschter und vielleicht schädigender Streustrahlung zu minimieren. 


\section{Literatur}

${ }^{1}$ Ewen K. Moderne Bildgebung. Thieme 1998

2 Fuchs M, Modler H, Schmid A, Stürmer KM. Strahlenschutz im Operationssaal. Oper Orthop und Traumatol. 1999; 11: $328-33$

3 Jansing PJ, Ewen K. Aktuelle Entwicklungen im Strahlenschutz bei beruflich Exponierten. Dtsch Med Wochenschr 2003; 128: $326-329$

4 Internationale Strahlenschutzkommission. Strahlenschutz und Sicherheit in der Medizin. ICPR-Veröffentlichung 73. Wirtschaftsverlag/Verlag für neue Wissenschaft, Bremerhaven 1999
${ }^{5}$ Klimpel H, Kreienfeld H, Kraus G. Strahlenschutz und Röntgendiagnostik. In: Tscherne $\mathrm{H}$, Regel, G. Trauma-Management. Springer 1997

${ }^{6}$ Verordnung über den Schutz vor Schäden durch ionisierende Strahlen (Strahlenschutzverordnung - StrSchV), vom 20.07.2001. BGBI. I S.1714 2002 I S.1459), geändert durch Artikel 2 der Verordnung vom 18. Juni 2002 (BGBl. I S.1869,1903)

7 Verordnung über den Schutz vor Schäden durch Röntgenstrahlen (Röntgenverordnung RöV), vom 8. Januar 1987. (BGBl. I S.114), in der Fassung der Bekanntmachung vom 30. April 2003 (BGBl. I S. 604)

8 Wich M, Spranger N, Ekkernkamp A. Intraoperative Bildgebung mit dem ISO $\mathrm{C}^{3}$. Chirurg 2004; 75: $982-987$

\section{Dr. med. Thomas Lein}

Ltd. Oberarzt, Strahlenschutzbeauftragter OP-Personal

Falko Moritz

Facharzt für Chirurgie

Dirk Lehmann

Facharzt für Diagnostische Radiologie Prof. Dr. med. Felix Bonnaire Chefarzt

Klinik für Unfall-, Wiederherstellungsund Handchirurgie

Krankenhaus Dresden-Friedrichstadt Städtisches Klinikum

Friedrichstr. 41

D-01067 Dresden 


\section{Buchbesprechungen}

Hofmann, G. 0.

Infektionen der Knochen und Gelenke

In Traumatologie und Orthopädie.

Urban \& Fischer, München,

1. Auflage 2004, $€ 82$.-,

ISBN 3-437-23400-5

Stoller D, Tirman P, Bredella M.

Bewegungsapparat -

Die 100 Top-Diagnosen.

Urban \& Fischer, München,

1. Deutsche Auflage 2004, $€$ 49,95,

ISBN 3-437-23460-9
Es wird ein Buch vorgestellt, das auf 274 Seiten mit je $80 \mathrm{~s} / \mathrm{w}$ und farbigen Abbildungen einen hervorragenden Überblick über diese Geisel auch der modernen Medizin gibt. In ausreichender Ausführlichkeit im Detail und doch kompakt ist es dem Autor gelungen alle wesentlichen, auf neuestem Stand befindlichen $\mathrm{Er}$ kenntnisse bezüglich Klassifikation, Diagnostik und Therapie darzustellen für: akute und chronische Osteitis, Gelenkund Endoprotheseninfektionen, hämatogene Osteomyelitis, Weichgewebs- sowie

Nach zwei englischsprachigen Auflagen aus den Jahren 2001 und 2002 erscheint nun die erste deutschsprachige Ausgabe des Taschenbuches „Bewegungsapparat - Die 100 Top-Diagnosen“. Angesprochen werden niedergelassene Ärzte und Ärzte in Weiterbildung, denen das Buch als kompaktes Nachschlagewerk im Kitteltaschenformat dienen soll. Bereits der Titel „100 Top-Diagnosen“ läßt schlußfolgern, daß es sich dabei keineswegs um eine vollständige Darstellung sämtlicher Erkrankungen des Bewegungsapparates handeln kann. Von namhaften amerikanischen Radiologen und Orthopäden werden in den ersten 6 Kapiteln traumatische und degenerative Läsionen der großen Gelenke beschrieben. Die Kapitel 7-9 befassen sich mit Erkrankungen des Knochenmarkes sowie mit Knochen- und Weichteiltumoren. Alle Krankheitsbilder werden streng nach dem gleichen Algo- spinale und paraspinale Infektionen. Wichtige zusätzliche Informationen erhält der Leser zum MRSA-Problem und zur Begutachtung. Weiterführende Studien werden durch die Angabe von Internetadressen sowie ein aktuelles Literaturverzeichnis ermöglicht. Der Preis ist angemessen, sodass dieses Buch hoffentlich den Weg nimmt, den es verdient : in die Hände aller operativ tätigen Unfallchirurgen und Orthopäden.

Karl Heinrich Winker

rhythmus abgehandelt, wobei jeder Entität eine anatomische Schemazeichnung oder ein MRT-Schnittbild vorangestellt ist. Erwartungsgemäß wird die Bildgebung differenziert beschrieben, so daß der klinisch tätige Arzt eine komprimierte Zusammenfassung der wesentlichen radiologischen Befunde erhält. Die Ausführungen zu Grundlagen, Pathologie, klinischem Bild und Therapie können bei der Kürze der Darstellung keineswegs dem Anspruch auf Vollständigkeit genügen. Der abschließende Hinweis auf 1 3 Literaturstellen ist ebenso unvollständig wie entbehrlich. $\mathrm{Zu}$ einem günstigen Preis angeboten könnte dieses Taschenbuch dem Kliniker ein schneller Ratgeber bei der Interpretation radiologischer Befunde sein.

Matthias Kunath, Erfurt 